\title{
The Ambivalent Mesenchyme-Pleomorphic Hyalinising Angiectatic Tumour
}

\author{
Anubha Bajaj* \\ Consultant Histopathologist, AB Diagnostics, India
}

*Corresponding author: Anubha Bajaj, Consultant Histopathologist, AB

Diagnostics, A-1, Ring Road, Rajouri Garden, New Delhi 110027, India.

Received Date: August 11, 2020

Published Date: September 03, 2020

\section{Introduction}

\section{Preface}

Pleomorphic hyalinising angiectatic tumour (PHAT) is an infrequently discerned, soft tissue, mesenchymal, spindle-shaped cellular neoplasm of uncertain cellular lineage initially scripted by Smith et al in 1996 and is contemplated as a neoplasm of intermediate malignant potential [1]. The exceptional, mesenchymal lesion is frequently cogitated within subcutaneous tissue and is composed of pleomorphic, spindle-shaped cells and ectatic blood vessels encompassed by hyalinised stroma and lack of mitosis [1,2].

Pleomorphic hyalinising angiectatic tumour can emerge as a component of collision tumour in combination with sarcomas such as myxofibrosarcoma. A myxoid, pleomorphic sarcoma can appear at the site of preceding pleomorphic hyalinising angiectatic tumour. Haemosiderotic fibrolipomatous tumour can be contemplated as a precursor lesion [1,2].

\section{Disease Pathogenesis}

Of obscure aetiology, classic pleomorphic hyalinising angiectatic tumour appears due to encroachment of developing neoplasm upon blood vessels of circumscribing, healthy soft tissue. Thereby, exuding plasma constituents of damaged endothelium precipitates a mechanism of neoplastic organization with characteristic emergence of perivascular hyalinization. Vascular alterations occur within a preliminary stage, prior to significant proliferation of spindle-shaped cells [2,3]. Released chemical products of vasoactive mast cells appear as a reaction to neoplasm- induced tissue injury which engenders enhanced vascular permeability during tumour genesis. Alternatively, it is posited that preliminary, developing pleomorphic hyalinising angiectatic tumour classically delineates a low to medium density of spindle-shaped cells, infrequent pleomorphic cells and myxoid, extracellular matrix. Aforesaid features configure a precursor lesion to classic pleomorphic hyalinising angiectatic tumour [2,3].

The neoplasm can also be derived from primitive, undifferentiated mesenchyme or stromal fibroblasts. Characteristic cellular pleomorphism arising within pleomorphic hyalinising angiectatic tumour is a delayed, degenerative phenomenon, pertaining to duration of lesion $[2,3]$.

\section{Disease Characteristics}

Adults are commonly implicated. Typically, pleomorphic hyalinising angiectatic tumour emerges within subcutaneous tissue of lower extremities although the lesion can also appear intramuscularly. The tumour is discerned upon the arms, forearm, shoulder, axilla, hand, upper extremities, trunk, thoracic region, buccal mucosa, oral cavity, buttock, scrotum, renal hilum, inguinal region, retroperitoneum, perineum or popliteal fossa. Commonly, pleomorphic hyalinising angiectatic tumour is located upon the leg (18.6\%), thigh(17.5\%), foot $(14.4 \%)$ and ankle $(10.3 \%)$ $[3,4]$. The gradually progressive neoplasm classically arises in adult population within an age range of 10 years to 89 years and a median age of tumour emergence at 57 years. Although devoid of racial predilection, the neoplasm demonstrates a slight female preponderance with a female to male proportion of $4: 3[3,4]$. Biological behaviour, tumour magnitude and duration of lesion is variable and pertains to tumour location. An incidental, enlarged mass can be discerned within deep-seated locations as the pelvic 
retroperitoneal space with abundant, loosely configured soft tissue. As the neoplasm is locally aggressive and delineates an estimated localized tumour reoccurrence of $33 \%$ to $50 \%$, the tumefaction is contemplated as a mesenchymal tumour of intermediate malignancy. Although distant metastasis is absent, pleomorphic hyalinising angiectatic tumour can occasionally reappear as a sarcoma [4].

\section{Clinical Elucidation}

Typically, tumour evaluation is achieved by radiography of incriminated region followed by contrast- enhanced magnetic resonance imaging (MRI) and accrual of a cogent tissue specimen. Tumour mass is irregular, localized, gradually progressive, abundantly vascular and firmly adherent to adjacent soft tissue with an absence of infiltration [4,5]. The neoplasm depicts a well demarcated, infiltrative perimeter. Prominent, ectatic, arterial blood vessels infuse tumour periphery and parenchyma. Although pleomorphic hyalinising angiectatic tumour depicts minimal mitosis and an absence of distant metastasis, aggressive localized tumour reoccurrence can incur significant morbidity. Therefore, the neoplasm is contemplated to be a borderline, intermediategrade, soft tissue sarcoma, rather than a benign neoplasm $[4,5]$.

\section{Histological Elucidation}

Grossly, a partially or predominantly cystic, elliptical to spheroidal, lobulated tumour mass of hues varying from tan to maroon is discerned with associated bruising, haematoma or infrequent necrosis. The neoplasm can be painless, painful or tender to touch. The tumefaction is un-encapsulated with an infiltrative perimeter. Several blood vessels traverse tumour surface. Tumour tissue is solid and of brittle texture. Cut surface is greyish/white and demonstrates foci of necrosis and vascular thrombosis $[4,5]$. On cytology, bland and enlarged, pleomorphic cells are admixed with hyalinised blood vessels. Tumour nodules are lobulated, spherical or elliptical. The tumefaction is non encapsulated, adheres to adjacent soft tissue and assimilates circumscribing soft tissue into the neoplasm [5]. Certain tumours depict an attenuated, complete capsule with smooth, clear tumour boundaries. Adipose tissue enveloping the lesion enunciates distinct margins. However, tumour confined to extremities is circumscribed by skeletal muscle and appears deficient in adipose tissue [5]. Microscopically, clusters of distended, thin walled, blood vessels with prominent hyalinization of vessel wall are delineated. Deposition of perivascular and intercellular ground substance ensues. Tumour cells are pleomorphic and disseminated amidst dilated blood vessels and foci of haemorrhage. Mitotic figures are exceptional or absent and minimal mitosis of up to 3 per 10 high power fields can be encountered. Atypical spindle-shaped cells incorporate hemosiderin with intra-nuclear and cytoplasmic inclusions and simulate cells of pleomorphic malignant fibrous histiocytoma (MFH) or Schwannoma. Enlarged, ectatic vascular spaces imbued with blood and fibrin are observed along with foci of perivascular hyalinisation. Mast cells are abundant whereas adipose tissue fragments are occasional [5,6]. Clusters of ectatic blood vessels are enveloped by fibrin- rich, hyaline substance and a peripheral cuffing of collagen lamellae which extend into the tumour mass and encircle individual cells, thus compartmentalising hyalinised material [6]. As the neoplasm is composed of spindle-shaped or spheroidal, plump pleomorphic cells, cellular or nuclear hyperchromasia, pleomorphism and lack cytoplasmic differentiation is noted. Cells incorporate hemosiderin along with cytoplasmic intermediate filaments vimentin and nuclear inclusions [6]. The circumscribed tumour imbued with thin walled, ectatic, hyalinised blood vessels is denominated with focal areas of haemorrhage and vascular thrombosis. Intermediately dense tumefaction with bland, elliptical cells, spherical nuclear contour and occasional, miniature nuclei can be observed. An admixture of hemosiderin- laden macrophages with disseminated, enlarged, atypical cells containing singular or multiple nuclei is enunciated. Foci of myxoid stroma are prominent. Necrosis is absent [5,6]. Aggregates of thin walled, ectatic blood vessels of variable magnitude are infiltrated and enveloped by amorphous, fibrin- rich, hyaline substance and focal, myxoid change. Characteristic angiectatic blood vessels are encompassed by sheets or fascicles of plump, spindle- shaped or spherical pleomorphic cells. A subset of cells abutting blood vessels are imbued with intracytoplasmic deposits of hemosiderin pigment. Pleomorphic cellular population is comprised of enlarged, singular cells intermixed with multinucleate cells with abundant cytoplasm, enlarged, irregular nuclei and occasionally, enlarged intra-nuclear inclusions [5,6]. Spindle-shaped neoplastic cells can be sparsely intermixed with hyalinised blood vessels. Predominantly bland, spindled cellular component is intermingled with scattered, enlarged atypical cells along with hemosiderin-laden macrophages [6]. Pleomorphic hyalinising angiectatic tumour is infiltrated with a mixed, chronic inflammatory cell exudate composed of mast cells, mature lymphocytes, plasma cells and eosinophils. Tumour mass exhibits a peripheral, partially myxoid, spindle-shaped, cellular component which can be contemplated as a precursor lesion to classic, pleomorphic hyalinising angiectatic tumour and is discerned within preliminary lesions [5,6] (Figure 1-7).

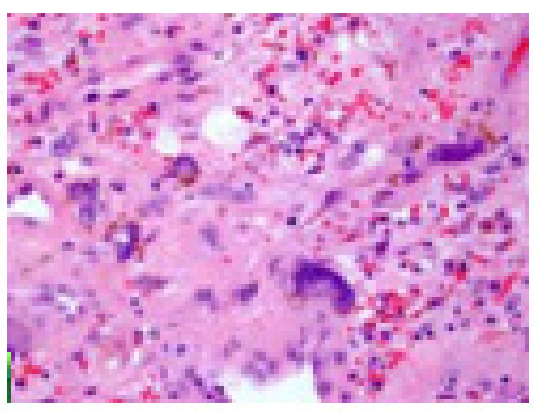

Figure 1: Pleomorphic hyalinising angiectatic tumour demonstrate clusters of attenuated blood vessels intermixed with thrombotic vasculature and chronic inflammatory cells interspersed within a fibrinous, myxoid stroma [9]. 


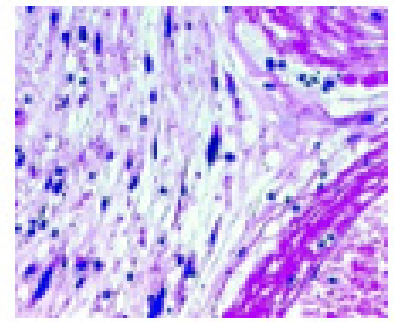

Figure 2: Pleomorphic hyalinising angiectatic tumour enunciating hyalinised blood vessels and intermingled pleomorphic spindle cells with minimal mitosis [10].

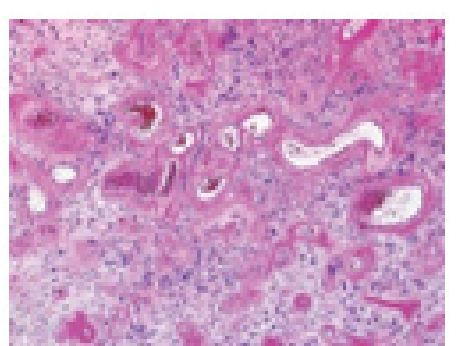

Figure 3: Pleomorphic hyalinising angiectatic tumour depicting several hyalinised blood vessels, myxoid stroma and a pleomorphic spindle to round cells [11].

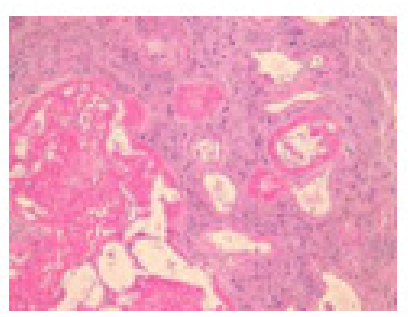

Figure 4: Pleomorphic hyalinising angiectatic tumour exemplifying numerous hyalinised blood vessels intermingled with pleomorphic, spindle cells and a fibrin-rich myxoid stroma with few mast cells [12].

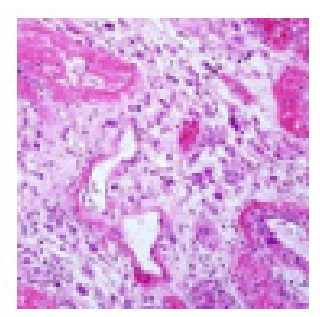

Figure 5: Pleomorphic hyalinising angiectatic tumour enunciating numerous hyalinised blood vessels, pleomorphic spindle-shaped cells and a fibrinous, myxoid stroma [13].

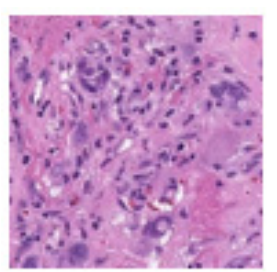

Figure 6: Pleomorphic hyalinising angiectatic tumour depicting pleomorphic, round cells, myxoid, fibrinous stroma and circumscribing hyalinised blood vessels [14].

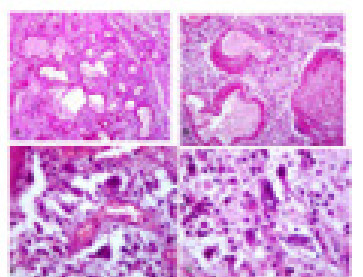

Figure7: Pleomorphichyalinising angiectatic tumour demonstrating foci of plump, pleomorphic, spindle cells encompassed in a fibrinrich stroma and hyalinised, ectatic blood vessels [15]

\section{Immune Histochemical Elucidation}

The neoplasm is immune reactive to CD34, vimentin, B- cell lymphoma 2 (bcl-2), vascular endothelial growth factor (VEGF), CD99 and focally immune reactive to Factor XIIIa, CD117 and cytokeratin(2). The tumour is immune non-reactive to S100 protein, CD31, CD68, von Willebrand factor, smooth muscle actin (SMA), desmin, epithelial membrane antigen (EMA) and mouse double minute 2 homolog (MDM2). Proliferation labelling index $\mathrm{Ki}-67$ is around $2 \%$ to $5 \%$, especially within proliferative zones, thus indicating a minimal presence of mitotically active cells. Immune reactivity to CD34 and vimentin indicate a mesenchymal cellular lineage. Immune non-reactive S100 protein eliminates a Schwannoma, a lesion commonly necessitating differentiation $[2,3]$. Pleomorphic hyalinising angiectatic tumour is also immune non-reactive to CD56, anaplastic lymphoma kinase 1(ALK-1), actin and anoctamin1 (DOG-1), thus distinguishing the neoplasm from adjunctive neurogenic or myogenic neoplasia or gastrointestinal stromal tumour. Perl's Prussian blue staining demonstrates iron deposition within the lesion. On cytogenetic analysis, the tumefaction reveals an unbalanced genomic translocation within chromosome 1 and 3 or chromosome 1 and 10 with genetic breakpoints confined to transforming growth factor- $\beta$ receptor 3 (TGFBR3) and meningioma- expressed antigen 5 (MGEA5) [2,3]. Pleomorphic hyalinising angiotectatic tumour demonstrates unbalanced genomic translocations involving chromosome 1 and 3 along with chromosome 1 and 10 with a karyotype of 45XX $\operatorname{der}(1) t(1 ; 3) p(31 ; q 12),-3, \operatorname{der}(10) t(1 ; 10)(p 31 ; q 25)$ [11]/46, XX4 $[2,3]$. Subsequent analysis with fluorescent in situ hybridization (FISH) and single nucleotide polymorphism analyses of certain tumefaction exemplifies demonstration of characteristic TGFBR3 and MGEA5 genetic rearrangements [2,3].

\section{Differential Diagnosis}

Pleomorphic hyalinising angiectatic tumour requires segregation from associated vascular and hyper-vascular neoplasia such as solitary fibrous tumour (SFT), para-ganglioma, gastrointestinal stromal tumour (GIST) and primary cutaneous myxofibrosarcoma [7]. Pleomorphic hyalinising angiectatic tumour necessitates a histological separation from neurilemmoma, undifferentiated pleomorphic sarcoma (UPS) and giant cell angiofibroma [7]. Pleomorphic hyalinising angiectatic tumour 
depicts an absence of immune reactive S100 protein, lack of tumour encapsulation and absence of Antoni A and Antoni B areas, in contrast to neurilemmona. Neurilemmoma lacks cellular and nuclear atypia, tumour is devoid of infiltrative capacity and is intensely immune reactive to S100 protein. Pleomorphic hyalinising angiectatic tumour delineates infrequent mitotic figures and presence of nuclear inclusions, in contrast to undifferentiated pleomorphic sarcoma $[7,8]$. Pleomorphic hyalinising angiectatic tumour demonstrates clusters of thin walled blood vessels, atypical cells with bland cytoplasm and intra-nuclear inclusions, in contrast to solitary fibrous tumour [8]. Solitary fibrous tumour is well defined and demonstrates intense, heterogeneous image enhancement which persists during delayed phase. Foci of necrosis, haemorrhage or cystic change are exemplified. Solitary fibrous tumour demonstrates an immune profile akin to pleomorphic hyalinising angiectatic tumour as the dual neoplasms exemplify vimentin, CD34 and CD99. Nevertheless, the neoplasms (SFT and PHAT) are histologically diverse [7,8]. Paraganglioma can enunciate a well-defined, hyper-vascular tumefaction and appear along the course of prominent blood vessels. With radiographic imaging, the tumefaction is heterogeneous and depicts foci of calcification. Upon magnetic resonance imaging (MRI), neoplasm depicts minimal signal intensity upon T1 weighted imaging and enhanced signal intensity upon T2 weighted imaging with significant image enhancement. Signal intensity of pleomorphic hyalinising angiectatic tumour usually displays intense arterial enhancement of magnitude greater than as discerned with solitary fibrous tumour or gastrointestinal stromal tumour and is diverse from paraganglioma $[7,8]$. Primary gastrointestinal stromal tumour (GIST) appears as enlarged, hypervascular, enhancing nodule with frequent foci of haemorrhage, necrosis or cystic degeneration [8]. Hyper-vascular alveolar soft part sarcoma (ASPS) necessitates a demarcation from pleomorphic hyalinising angiectatic tumour. On contrast- enhanced imaging, the neoplasms (ASPS and PHAT) delineate identical manifestations whereas alveolar soft part sarcoma exhibits an enhanced signal intensity upon T1 and T2 weighted imaging. Malignant fibrous histiocytoma (MFH) is occasionally immune reactive to CD34 and is immune non-reactive to CD99 [8].

\section{Investigative Assay}

Radiographic features of pleomorphic hyalinising angiectatic tumour are nonspecific and inadequately defined. However, typically a soft tissue mass with an absence of osseous alterations or calcification is delineated [7]. On radiographic imaging, pleomorphic hyalinising angiectatic tumour requires a segregation from subcutaneous malignant metastasis, malignant melanoma or soft tissue sarcoma. Monitoring with serial radiographic images demonstrates a tendency for localized reappearance [7]. On ultrasonography, a hypoechoic lesion with an intrinsic hyperechogenicity and characteristic hyperechoic tissue interrupted with presumably vascular, hypoechoic bands is exemplified [8]. Computerized tomography (CT) and magnetic resonance imaging
(MRI) demonstrates a well-defined tumefaction with homogenous, dense signal intensity. Foci of elevated density are detected upon non-contrast computed tomography (CT), with an absence of image enhancement, on account of intra-lesional haemorrhage [8]. On contrast- enhanced imaging, the tumour is prominent, well defined, heterogeneous and enunciates several circumscribing dilated blood vessels. Magnetic resonance imaging (MRI) delineates a soft tissue mass with accompanying oedema or haematoma. The neoplasm is hypo-intense to isointense upon T1 weighted sequences, heterogeneously isointense to hyper-intense on T2 weighted sequences and exhibits heterogeneous enhancement with intravenous administration of gadolinium-based contrast [8]. Haemorrhage within the tumefaction can be confirmed with computerized tomography (CT), magnetic resonance imaging (MRI), intra-operatively and on histological examination. Tumour haemorrhage is contingent to abundant vascularity and magnitude of the tumefaction. Enlarged lesions are liable to bleed. Besides necrosis and haemorrhage, irregular foci with an absence of obvious enhancement are discerned. Nodules confined to the neck or renal hilum display clear outlines. However, tumefaction confined to extremities is ill- defined [8]. Positron emission tomography (PET CT) demonstrates a mildly hypermetabolic lesion with an absence of hypermetabolic tumour metastasis. A well-defined tumour mass with an absence of fluid on fluoro-deoxy glucose (FDG) uptake is discerned, concurrent with minimal mitosis within the neoplasm $[7,8]$.

\section{Therapeutic Options}

The neoplasm is subjected to radical surgical excision. As the nodule is imbued with distended blood vessels, preoperative embolization can significantly reduce intraoperative haemorrhage and ensure safe surgeries [8]. As a capacity for localized tumour reoccurrence is demonstrated, surgical extermination with a wide margin of normal, tumour-free tissue perimeter and extended monitoring are optimal therapeutic approaches. Majority of neoplasms are subjected to marginal resection [8]. Surgical extermination of the neoplasm can be combined with administration of low dose radiation in order to decimate localized tumour relapse. Adjuvant radiotherapy is employed to decimate proportionate, localized tumour reoccurrence, although it may not be beneficial. Chemotherapy is devoid of cogent contribution while treating the neoplasm on account of absence of distant metastasis $[7,8]$.

\section{Acknowledgement}

None.

\section{Conflicts of Interest}

No conflicts of interest.

\section{References}

1. Smith ME, Fisher C (1996) Pleomorphic hyalinising angiectatic tumour of soft parts-a low grade neoplasm resembling neurilemmoma. Am J Surg Pathol 20(1): 21-29. 
2. Folpe AL, Weiss SW (2004) Pleomorphic hyalinising angiectatic tumour : analysis of 41 cases supporting evolution from a distinctive precursor lesion. Am J Surg Pathol 28(11): 1417-1425.

3. Chu ZG, Liu MQ (2016) Pelvic retroperitoneal pleomorphic hyalinising angiectatic tumour (PHAT) of soft tissue- a case report. BMC Med Imaging 16: 28.

4. Jaramillo CJ, Wojcik J, Kristy Weber, Ronnie Sebro (2018) Imaging and histological appearance of pleomorphic hyalinising angiectatic tumoura case series and literature review Oncol Lett 15(4): 4720-4730.

5. Balasubiramaniyan V, Muduly D, Anita P Javalgi, Shefali Goyal (2019) Pleomorophic hyalinising angiectatic tumour of the mesorectum- a rare case and review of literature Autops Case Rep 9(3): e2019097.

6. Carter JM, Sukov WR, (2014) TGFBR3 and MGEA5 rearrangements in pleomorphic hyalinising angiectatic tumours and the spectrum of related neoplasms. Am J Surg Pathol 38(9): 1182-1192.
7. Brazio PS, Morrison AL (2016) Large pleomorphic hyalinising angiectatic tumour of the forearm-a multidisciplinary perspective. Surgery 159 : 1471-1473.

8. Kane PM, Gaspar MP (2016) Pleomorphic hyalinising angiectatic tumour arising in the hand- a case report. Hand (N.Y) 11: NP20-NP23.

9. Image 1 Courtesy: UPOJ.com.

10. Image 2 Courtesy: Pathology outlines.

11. Image 3 Courtesy: Basic Medical Key.

12. Image 4 Courtesy: Dermnet. NZ.com.

13. Image 5 Courtesy: Semantic Scholar.

14. Image 6 Courtesy: Twitter.com.

15. Image 7 Courtesy: OA Publishing London. 